\title{
Cognitive Maps in the Development of the Multi- dimensional System of Objects' Management for the Manufacturing of Products From Composites
}

\author{
Tatiana Sazonova \\ Kumertau branch of \\ Orenburg State University \\ Kumertau, Russia \\ t.sazonowa@yandex.ru
}

\author{
Larisa Polyakova \\ Kumertau branch of \\ Orenburg State University \\ Kumertau, Russia \\ lara_umnr@kfosu.edu.ru
}

\begin{abstract}
The article discusses the problem of automation of technological processes for the manufacturing of products from polymer composite materials used in mechanical engineering. Classification of control systems is represented according to the degree of completeness of a priori information about the control object and the complexity of the system's structural scheme. The structure of the automation of technological process is given using the example of the manufacture of composite materials. This structure includes the production of the binder, prepreg, polymerization, drying, gluing and information field. The methodology of cognitive maps modeling in the development of the multidimensional system of objects' management for the manufacturing of products from composites is considered. control systems based on cognitive maps, implemented on clear terms, are closer to human thinking and natural language and allow build a control algorithm adequate to the real production process. Cognitive maps based on cognitive modeling allow the use of incomplete, fuzzy and even contradictory information from a subject expert about a complex object, including polymerization. The development of control systems for the manufacturing of polymer composite materials at the heat treatment stage based on the cognitive maps on clear terms is an urgent scientific problem. In addition, the solution of it will allow significantly increase productivity, reduce energy consumption and improve the quality of materials subjected to
\end{abstract} heat treatment.

Keywords - cognitive maps, technological process, composite materials, modeling, automation

\section{INTRODUCTION}

Currently the efforts of many scientists are aimed at developing new automated or automatic methods for controlling production processes in mechanical engineering. The transition to new methods of managing the manufacturing of products from polymer composite materials is directly related to the technology of its manufacturing and the level of automation. A systematic approach to the consideration of various processes is one of the most important requirements of the modern stage of scientific and technological progress. Therefore, it should find application in the study of all issues related to the development and improvement of management processes for all departments of the enterprise.

\section{THE THEORETICAL BASIS}

In consideration of different management systems they must be classified. According to the constructs of the management theory, two main features may be chosen to classify them properly. The first of them is the degree of completeness of a priori information about the control object, and the second is the complexity of the system's structural scheme. In turn, according to the first feature, systems are divided into systems with a priori information specified in the form of probability-theoretical characteristics and systems with incomplete a priori information. As for the second feature, the systems can be multidimensional mutually adjustable, adjustable with a variable structure, closed with a hierarchical control structure and systems of arbitrary complexity. In addition to this classification, systems can be classified by area of application: these are technical, economic, information and other systems. Despite the variety of complex systems, the most typical features include:

- the presence of subsystems with pronounced local properties;

- the presence of a hierarchy in the management structure of the system;

- the existence of a global criterion that is optimal for the system as a whole;

- communication between local subsystems, carried out not only directly, but also through control signals that are generated based on the dependence of the global criterion on the local one;

- the presence of two mutually affecting flows: material and information;

- symbiosis of man and machine in the system.

The technological process for the manufacturing of products from polymers can be represented as a megasystem that includes several hierarchical systems (Fig. 1).

This mega-system is a tree-like structure, where the manufacture of products from composites forms the root peak [1]. A distinctive feature of this megasystem is that its structure is a collection of parallel operating subsystems of a sufficiently high level and having various degrees of significance. So, for example, at the root vertex level, the control component is dominant, because at this level, strategic decisions are made that can affect the automation of technological processes (ATP) of the entire industry as a whole. And at the lowest level, management decisions are made only within the framework of specific operations and affect mainly their efficiency. 


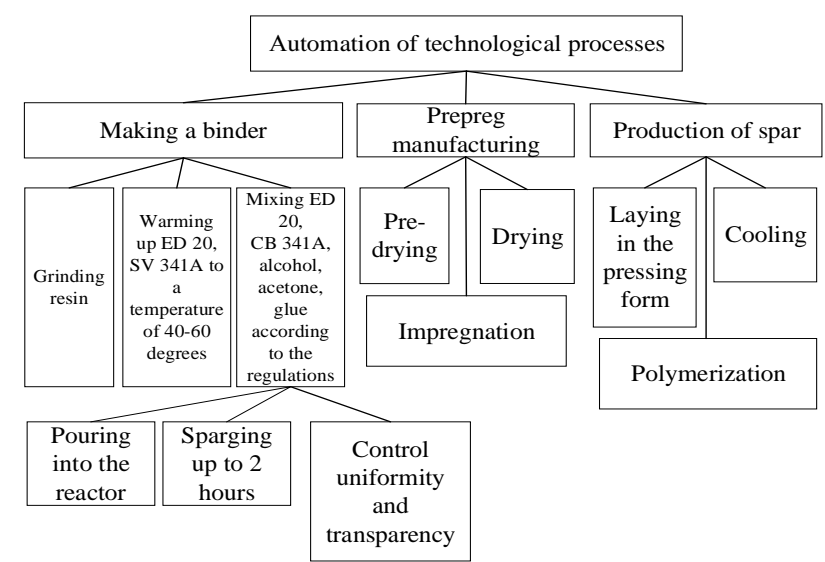

Fig. 1 - System for the technological production of products from polymer composite materials

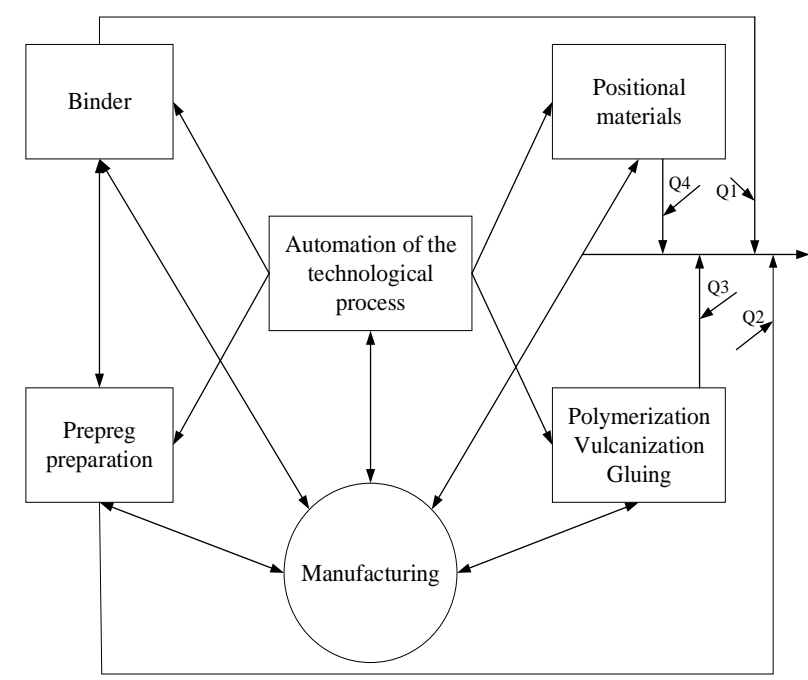

Fig. 2 - The structure of ATP products of polymer composite materials

According to Figure 2, the ATP for the production of composite materials includes a number of subsystems, such as the binder, prepreg, polymerization, drying, bonding, and information field subsystems. Each subsystem brings its own effectiveness value Q1, Q2, Q3, Q4 to the overall effective operation of the system. In turn, each of these subsystems includes subsystems of an even lower level. And these subsystems have their own characteristics and tasks, and at the same time they all work together as they are created and operate to achieve the general goal of the enterprise - to obtain the maximum quantity and quality of helicopter products. So in the ATP system there are subsystems for the processes of preparing a binder, prepreg, and polymerization. Each of the automated control systems (ACS) can be considered as an independent system for ensuring the production cycle. The structure of the polymer composite materials manufacturing in engineering is shown in Fig. 3. This structure has a complex system of ties and in general it does not meet the technical conditions for the production of products from composite materials.

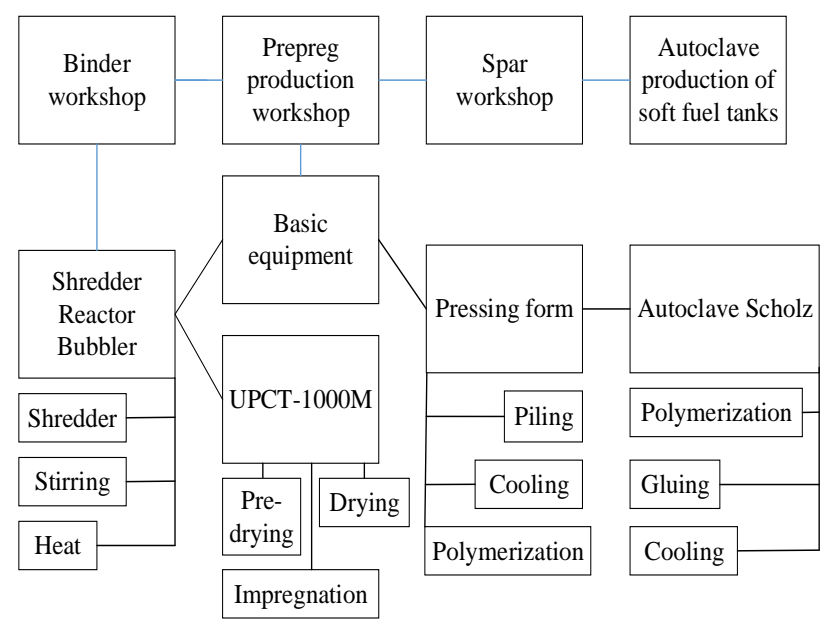

Fig. 3 - Structure of automated technological production of products in mechanical engineering

As at present all components of this structure have undergone significant changes, which has resulted in the elimination of some parts of the technical process and their reorientation to other types of operations. In addition, there are problems with the availability of qualified specialists. In this regard, it is necessary to carry out structural transformations, all ATP units from manufacturers to consumers, including auxiliary industries, such as technical services, which include the acquisition, use and operation of mobile energy facilities. Particular attention should be paid to information support in order to transfer the experience of experts to build modern intelligent control systems $[2,3,4]$.

An expert and analyst have to rely on their own experience and intuition as they make decisions in the conditions of a complete or partial lack of quantitative information. They use a subjective model based on expert assessments of analysts to make decisions as a model of a dynamic situation, i.e. on knowledge. The subjective model of a poorly structured dynamic situation is called a cognitive map $[5,6]$.

\section{Modeling Methodology}

The activity of experts and analysts aimed at studying the situation and making decisions using cognitive maps is a methodology of a logical-temporal structure $[6,7,8]$. It includes applying various methods and techniques: constructing a cognitive map, its parameterization, obtaining forecasts of situations, verification, cognitive map adjustments and decision making.

At the moment, a fairly large variety of simulation is presented in a variety of subject areas. More than 50 software products are presented on the market for simulation [9].

All of them work with numerical data characterizing the simulated system, and simulation models of systems are built in the framework of the concept of analysis of "hard" systems $[9,10]$.

To computer simulation systems knowledge working in the framework of the concept of "soft" system analysis, can be attributed expert systems that model experts' knowledge and behavior in solving problems. For the solution of these problems considerable experience in decision making has 
been accumulated. However, this experience is difficult to formalize in framework of the "rigid" systems theory.

Computer systems for modeling cognitive maps form a special class of simulation systems, which, according to the functionality and organization principles, can be positioned between simulation systems and expert systems. In terms of software architecture, cognitive map modeling systems inherit the properties of simulation systems of "rigid" systems in organizing simulation processes and expert systems in extracting and presenting expert knowledge, as well as its processing.

Thus, the problem of controlling complex processes in technological plants, especially at the heat treatment stage in autoclaves, is that they belong to the class of multiply connected, inertial, non-stationary and non-linear with distributed parameters of control objects [11]. Nevertheless, the regulation of the parameters characterizing the process is usually carried out by one-dimensional proportionalintegral-differential (PID) -regulators. They represent linear controls without compensating for the mutual influence of the controlled parameters. Hence, the poor quality of management and, as a result, an increase in the share of defective products in the finished product and unjustified energy costs.

An analysis of the works devoted to the automation of complex processes in a number of industries shows that the potentialities of multidimensional PID and fuzzy controllers (FC) in reduction the error of regulation and compensation for the mutual influence of control loops in the mentioned control systems are practically exhausted. Therefore, improvement of finished products' quality and reduction of heat energy losses are also exhausted. In accuracy, the logical control implemented on FC is inferior to the PID control of processes not only in nonlinear, but also in linear multiply connected control objects. Therefore, there was a need to develop concepts, models, methods and algorithms for the logical control of these processes in production, allowing improving the quality of process control by translating the experience and knowledge of domain experts into the correct control algorithms and programs [9].

The complexity of technological processes does not allow interpreting them in the form of a reliable and adequate mathematical model, therefore, control in such cases is performed using typical fuzzy controllers (TFC). Control systems for complex technological processes using TFC are duly reflected in the works of L.A. Zade, E.A. Mamdani, M. Sugeno, S. Osovsky, and others. However, the vast majority of the observed low accuracy of the simulation, which is of a qualitative nature. For this reason, there is a decrease in consumer properties of the finished product, as well as an overexpenditure of raw materials and energy in industrial process plants.

Unlike typical fuzzy regulators (TFR), fuzzy regulators with interval uncertainty (FR with IU), including multidimensional (MFR with IU), control systems based on cognitive maps, implemented on clear terms, are closer to human thinking and natural language and allow build a control algorithm adequate to the real production process.

The above arguments, as well as the insufficient knowledge of cognitive maps implemented on clear terms as part of control systems for multiply connected objects, suggest that the development of control systems for the manufacturing of polymer composite materials at the heat treatment stage based on the cognitive maps on clear terms is an urgent scientific problem. In addition, the solution of it will allow significantly increase productivity, reduce energy consumption and improve the quality of materials subjected to heat treatment.

Cognitive maps based on cognitive modeling [4,5,6,7] allow the use of incomplete, fuzzy and even contradictory information from a subject expert about a complex object. And in this case, the heat treatment process in an autoclave to control it. Cognitive maps make it possible to determine the most significant and significant relationships between factors [2,8] affecting the polymerization process in an autoclave. As well as they indicate the point of application of the control action of the required size, shape and sign in order to obtain the desired results $[3,12]$.

Concepts in a clear cognitive map, as well as in a fuzzy one, can take values from the range of real numbers $[0,1]$.

The term "clear" means that the process of regulating a given parameter (pressure and temperature) occurs in a welldefined range of experts.

For the logical control of the stages of purge and rise, the temperature in the autoclave during the manufacture of the prepreg is represented by a set of clear terms $\left(\mathrm{T}_{\mathrm{T} 1} \div \mathrm{T}_{\mathrm{T} 8}\right)$.

By their logical nature, terms $\left(\mathrm{T}_{\mathrm{T} 1} \div \mathrm{T}_{\mathrm{T} 8}\right)$ are arguments of two-valued logic. Their value on the universal numerical axis is described by the following expressions:

where $G S=G S_{1} \& G S_{2} \& G S_{3} \& G S_{4}$ - is the conjunction of track sensors monitoring the closed position of the autoclave covers.

$$
\begin{aligned}
& T_{T 1}=\left\{\begin{array}{l}
1, \text { If } G S \cdot\left(20^{\circ} \mathrm{C} \leq T \leq 50^{\circ} \mathrm{C}\right) ; \\
0, \text { If }\left(T>50^{\circ} \mathrm{C}\right) \cdot \overline{G S}
\end{array}\right. \\
& T_{T 2}=\left\{\begin{array}{l}
1, \text { If } G S \cdot\left(50^{\circ} \mathrm{C}<T \leq 70{ }^{\circ} \mathrm{C}\right) ; \\
0, \text { If }\left(T>70{ }^{\circ} \mathrm{C}\right) \cdot \overline{G S} ;
\end{array}\right. \\
& \text {... } \\
& \text {.. } \\
& T_{T 7}=\left\{\begin{array}{l}
1, \text { If } G S \cdot\left(150^{\circ} \mathrm{C}<T \leq 170^{\circ} \mathrm{C}\right) ; \\
0, \text { If }\left(T>170^{\circ} \mathrm{C}\right) \cdot \overline{G S} ;
\end{array}\right. \\
& T_{T 8}=\left\{\begin{array}{l}
1, \text { If } G S \cdot\left(170^{\circ} \mathrm{C}<T \leq 190^{\circ} \mathrm{C}\right) ; \\
0, \text { If }\left(T>190^{\circ} \mathrm{C}\right) \cdot \overline{G S} .
\end{array}\right.
\end{aligned}
$$

From the expressions (1), the most important property of the set of clear terms follows, which allows several times to increase the speed of clear logical controllers, since at any given time only one clear term has a value equal to a logical unit. As an example, Figure 4 shows the logical diagram of the algorithm for working out the following production rule If $\mathrm{T}_{5}=\left(\bar{T}_{52-} \cdot X_{1} \cdot \bar{Y}_{2}+\bar{T}_{53-} \cdot X_{2} \cdot Y_{3}\right) \cdot T_{51 \text {, }}$, that $F_{5}=F_{51-}$,

Where $T_{52-}, T_{53-}-$ second and third clear terms of the first control loop MFR with IU;

$X_{1}, X_{2}$ - discrete input;

$Y_{2}$ и $Y_{3}$ - autoclave output variables. 


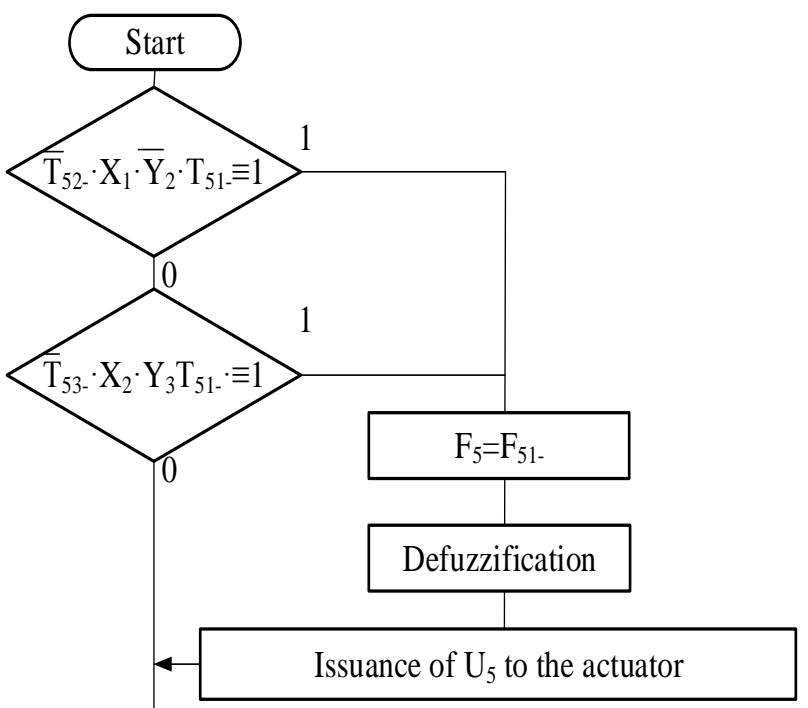

\section{Ending}

Fig. 4 - Software implementation of the production rule (1)

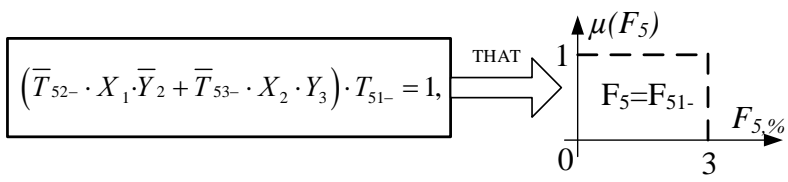

Fig. 5 - Scheme of working out the production rule (1)

Since the conditional part of rule (1) is a function of two-valued logic, it is legitimate to use the means of Boolean algebra to synthesize and minimize its structure (synthesis of logical functions by their unit values or using sequential equations, minimization of two-valued logic by the Quine-Mac-Claski method, etc.). This helps to reduce the time and improve the quality of design MFR with IU. In addition, regardless of the complexity of the structure of the antecedent of the production rule, the result of its scanning is one of the terms of adjustable size (Figure 5), the width of which will determine the error of regulation. Since the minimum width of a clear term is determined by the resolution of the programmable controller on which the MFR with IU is implemented, it becomes clear that there are practically unlimited possibilities for reducing the regulation error.

\section{CONCLUSION}

In this article a systematic approach to the process of development of ATP, the role and place of information support in ATP, forecasting issues in the decision making process of creating ATP, the role of cognitive maps' modeling in the development of a multidimensional system of objects' management for the composites manufacturing during design ATP are considered and analyzed.

\section{REFERENCES}

1. Polyakova L.Yu. Management of the technological process of manufacturing a prepreg for aircraft products // Abstract of dissertation for the degree of candidate of technical sciences: 05.13.06. - Ufa, USATU, 2007. (in Russian)

2. Kayashev A. I., Sharipov M. I., Muravyova E. A., Bagrov K. A. Cognitive modeling of petrochemical control objects with interpretation of concepts and the relationships between them by a set of clear terms // Proceedings of the XII All-Russian meeting on management problems of VSPU-2014 Moscow June 16-19, 2014 (in Russian)

3. Carvalho, J.P. Rule- based fuzzy cognitive maps and fuzzy cognitive maps - a comparative study // In Proc. of the 18th International Conference of the North American Fuzzy Information Processing Society, NAFIPS'99. - New York, 1999. - P. 115-119.5.

4. Ntarlas, O.D. Groumpos, P.P. A survey on Applications of fuzzy cognitive maps in business and management // Vestnik UGATU. -2014. -№ 5. - P. 3-7.

5. Borisov V.V., Fedulov A.S. Generalized fuzzy cognitive maps // Neurocomputers: development, application. 2004. No. 4. P. 3-20. (in Russian)

6. Kosko, B. Fuzzy Cognitive Maps// International Journa1 on Man-Machine Studies. 1986. Vol. 24. P. 65-75.

7. Tolmen, E.C. Cognitive maps in rats and men // Psychological Review. - 1948. - Vol. 42, № 55. - P. 189208.

8. Axelrod, R. Structure of Decision: the cognitive maps of political elites. - N. Y.: Prinston Univ. Press, 1976.

9. Kulinich, A.A. Modeling of dynamic processes in the conceptual system of the subject for the generation of creative solutions // Cognitive research: Sat. scientific papers. - M., 2006. - Issue. 1. - P. 94 -122. (in Russian)

10. Korostelev, D.A., Lageryov, D.G., Podvesovsky, A.G. The decision support system based on fuzzy cognitive models "IGLA" // The Eleventh National Conference on Artificial Intelligence with the international participation of KII - 2008, Dubna, September 28 - October 3, 2008 - M., 2008. - T. 3. - P. 327-329. (in Russian)

11. Sazonova, T.V. Bogdanov, A.V., Muravieva, E.A. Multidimensional clear logical regulator, with the development of production rules in situational subprograms // Bulletin of the Orenburg State University. - 2012. - No. 4. - P.248-252. (in Russian)

12. Prichina, O.S., Fros, D.L, Ruiz, K. The use of fuzzy cognitive maps for organizational diagnostics of an enterprise and modeling of activity // Bulletin of the Taganrog Institute. A.P. Chekhov. - 2014. - N. 1. - P. 3437. (in Russian) 